\title{
Lung injury and fibrosis induced by a mutant form of surfactant protein C
}

\begin{abstract}
Timothy S. Blackwell Nashville, Tennessee, USA. Department of Cell and Developmental Biology, Vanderbilt University School of Medicine, Nashville, Tennessee, USA.

Although mutant forms of the gene encoding surfactant protein $C$ (SFTPC) have been linked to interstitial lung disease, the mechanisms by which the most common of these mutations, SFTPC ${ }^{173 T}$, results in lung fibrosis are uncertain. In this issue of the $J C I$, Nureki et al. developed a knockin mouse model and showed that SFTPC ${ }^{173 T}$ is expressed by alveolar type II (AT2) epithelial cells in the lungs. These mice developed an agerelated fibrotic phenotype when the mutant allele was expressed at low levels and acute lung inflammation/injury followed by lung fibrosis when mutant SFTPC ${ }^{173 T}$ expression was enhanced. This work provides important information regarding the impact of AT2 cell dysfunction on fibrotic remodeling in the lungs.
\end{abstract}

Division of Allergy, Pulmonary and Critical Care Medicine, Department of Medicine, Vanderbilt University Medical Center, Nashville, Tennessee, USA. Department of Veterans Affairs Medical Center,

\section{Surfactant protein mutations and interstitial lung disease}

Although understanding of the genetic underpinnings of interstitial lung disease (ILD) is far from complete, identification of rare genetic variants that associate with disease in some families has provided valuable information regarding key cells and pathways that regulate fibrotic remodeling in the lungs. Outside of defined genetic disorders, such as Hermansky-Pudlak syndrome, surfactant protein C (SFTPC) was the first gene associated with ILD. Nogee and colleagues identified a heterozygous rare variant in this gene in an affected mother and child in 2001 (1). In 2002, a rare missense variant, $S F T P C^{L 188 Q}$, was shown to segregate with disease in a large family with ILD, including idiopathic pulmonary fibrosis (IPF) in affected adults (2). Subsequently, ILD-associated mutations in another surfactant protein, SFTPA2, were identified (3). Together, these studies provide convincing evidence that alveolar type II (AT2) cells, which produce surfactant proteins in the lungs, are inte- gral to disease pathogenesis. Rare variants that involve the $\mathrm{C}$-terminal portion (or BRICHOS domain) of proSP-C result in protein misfolding, aggregation, and endoplasmic reticulum (ER) stress (4-6); however, the mechanisms linking AT2 dysfunction and disease in individuals with the most common SFTPC mutation, SFTPC ${ }^{I 73 T}$, in humans with ILD have remained unclear. In this issue, Nureki et al. (7) use a clever strategy to develop a knockin mouse model to investigate how this toxic gain-of-function mutation causes AT2 dysfunction and leads to progressive lung fibrosis.

\section{Lung fibrosis development in SFTP [ $^{173 T}$-expressing mice}

Prior attempts to express mutant forms of SFTPC in AT2 cells in vivo have been hampered by perinatal mortality in response to constitutive expression of mutant SFT$P C$ during lung development and lack of a spontaneous phenotype following induction of low levels of SFTPC ${ }^{L 188 Q}$ in adult mice $(5,8,9)$. The strategy employed by

Related Article: p. 4008

Conflict of interest: TSB declares research support from Boehringer Ingelheim and Celgene.

Reference information: / Clin Invest. 2018;128(9):3745-3746. https://doi.org/10.1172/JCI122727.

Nureki et al. involves using a construct to insert an HA-tagged SFTPC ${ }^{I 73 T}$ mutant containing a PGK-Neo cassette in intron 1, which functions to limit expression of the mutant allele, into the endogenous Sftpc locus (7). Despite evidence of altered protein processing and trafficking, the resulting phenotype was relatively mild, with alveolar septal thickening and increased collagen content. Removal of the inhibitory PGK-Neo cassette via tamoxifeninduced expression of Flp-O recombinase enhanced SFTPC ${ }^{I 73 T}$ expression, resulting in embryonic lethality during the saccular stage of lung development, similar to what has been observed in previous models (8, 9). However, when the PGK-Neo cassette was removed after lung development, increased SFTPC ${ }^{I 73 T}$ expression in homozygous mice resulted in acute lung injury, with substantial mortality followed by a fibrotic ILD-like phenotype. Heterozygous SFTPC $C^{I 73 T /+}$ mice developed an intermediate phenotype. Intriguingly, while Nureki et al. found evidence of increased macroautophagy, they found no evidence of AT2 cell apoptosis in this model (7). These findings are consistent with prior studies in which SFTPC ${ }^{I 73 T}$ was overexpressed in vitro (10) but contrast with results from a variety of animal model studies that have linked AT2 cell injury and apoptosis to subsequent development of lung fibrosis $(5,6)$. Instead, AT2 cells expressing $S F T P C^{I 73 T}$ had increased levels of TGF- $\beta 1$, which can induce production of collagen and other matrix components by mesenchymal cells. Also, SFTPC ${ }^{I 73 T}$-expressing AT2 cells produced increased levels of CCL2, an important chemokine for recruitment of monocyte-derived macrophages, which were increased in SFT$P C^{I 73 T}$-expressing mice and have been shown to participate in fibrotic remodeling $(11,12)$. In addition to a persistent increase in macrophages in the lungs, an early increase in neutrophils followed by a transient eosinophil influx was identified 
after deletion of the PGK-Neo cassette (7). This inflammatory cell influx was accompanied by only a modest increase in cytokine levels in bronchoalveolar lavage and an absence of the classic Th2 cytokine response that has been linked to fibrosis in some models (13).

\section{Summary and future directions}

The prevailing paradigm to explain IPF pathogenesis suggests that recurrent micro-injuries in the alveolar compartment culminate in lung fibrosis. While alveolar injury has been thought to result from environmental stimuli, this study by Nureki et al. raises the possibility that focal alveolar injury might be driven by endogenous AT2 cell activation. Interestingly, lung injury was worse in male SFTPC ${ }^{I 73 T}$-knockin mice compared with females, potentially shedding light on the preponderance of male IPF patients. Further studies will be needed to better characterize the AT2 cell phenotype that drives alveolar capillary barrier dysfunction and inflammatory cell influx. Moreover, it will be important to determine whether inflammatory cells, particularly macrophages, are essential for development of fibrosis in this model.

Although substantial apoptosis of AT2 cells was not observed in conjunction with lung injury in SFTPC $C^{I 73 T}$-knockin mice, cleaved caspase- 3 was only measured at 2 and 4 weeks after tamoxifen treatment to remove the PGK-Neo cassette. Therefore, an early increase in AT2 cell apoptosis cannot be excluded as a possibility. Regardless, the total number of HA-tagged AT2 cells was increased by 7 days after tamoxifen treatment and persisted for up to 4 weeks. This finding is potentially relevant to human disease, as areas of AT2 cell hyperplasia are common in the IPF lung. The observed increase in AT2 cells in the alveolar compartment raises the question of whether there could be a defect in differentiation of AT2 cells to alveolar type I (AT1) epithelium in this model, which has been proposed in human IPF. Future studies to determine whether AT1 cell injury or death occurs in this model could be enlightening.

The SFTPC $C^{I 73 T}$-knockin mouse is the first model derived from an IPF-associated gene that develops spontaneous lung fibrosis. Other models with mutations in genes associated with familial IPF require a fibrotic stimulus, such as bleomycin, and manifest as increased fibrosis. Therefore, this model provides a translationally relevant platform to further explore disease pathogenesis and test potential therapeutic approaches. This work highlights an important strategy to operationalize genetic data by developing models wherein specific disease-associated mutations are expressed in appropriate cell types. This approach is particularly applicable in situations where the genetic variant (like SFTPC ${ }^{I 73 T}$ ) encodes a toxic gain-offunction protein.

\section{Acknowledgments}

TSB is supported by the NIH/National Heart, Lung, and Blood Institute (NIH-NHLBI; P01HL092870), the US Department of Defense, and the Department of Veterans Affairs.

Address correspondence to: Timothy S. Blackwell, Division of Allergy, Pulmonary and Critical Care Medicine, Vanderbilt University School of Medicine, 1611 21st Avenue South, T-1218 Medical Center North, Nashville, Tennessee 37232, USA. Phone: 615.322.3412; Email: timothy. blackwell@vanderbilt.edu.
1. Nogee LM, Dunbar AE, Wert SE, Askin F, Hamvas A, Whitsett JA. A mutation in the surfactant protein $\mathrm{C}$ gene associated with familial interstitial lung disease. N Engl JMed. 2001;344(8):573-579.

2. Thomas AQ, et al. Heterozygosity for a surfactant protein $\mathrm{C}$ gene mutation associated with usual interstitial pneumonitis and cellular nonspecific interstitial pneumonitis in one kindred. Am J Respir Crit Care Med. 2002;165(9):1322-1328.

3. Wang Y, et al. Genetic defects in surfactant protein $\mathrm{A} 2$ are associated with pulmonary fibrosis and lung cancer. Am J Hum Genet. 2009;84(1):52-59.

4. Kropski JA, Blackwell TS. Endoplasmic reticulum stress in the pathogenesis of fibrotic disease. J Clin Invest. 2018;128(1):64-73.

5. Lawson WE, et al. Endoplasmic reticulum stress enhances fibrotic remodeling in the lungs. Proc Natl Acad Sci USA. 2011;108(26):10562-10567.

6. Burman A, et al. Localized hypoxia links ER stress to lung fibrosis through induction of $\mathrm{C} / \mathrm{EBP}$ homologous protein. JCI Insight. In press.

7. Nureki SI, et al. Expression of mutant Sftpc in murine alveolar epithelia drives spontaneous lung fibrosis. J Clin Invest. 2018; 128(9):4008-4024.

8. Bridges JP, Wert SE, Nogee LM, Weaver TE. Expression of a human surfactant protein $\mathrm{C}$ mutation associated with interstitial lung disease disrupts lung development in transgenic mice. J Biol Chem. 2003;278(52):52739-52746.

9. Conkright JJ, Na CL, Weaver TE. Overexpression of surfactant protein-C mature peptide causes neonatal lethality in transgenic mice. Am J Respir Cell Mol Biol. 2002;26(1):85-90.

10. Mulugeta S, Nguyen V, Russo SJ, Muniswamy $\mathrm{M}$, Beers MF. A surfactant protein C precursor protein BRICHOS domain mutation causes endoplasmic reticulum stress, proteasome dysfunction, and caspase 3 activation. Am J Respir Cell Mol Biol. 2005;32(6):521-530.

11. Young LR, et al. Epithelial-macrophage interactions determine pulmonary fibrosis susceptibility in Hermansky-Pudlak syndrome. JCI Insight. 2016;1(17):e88947.

12. Larson-Casey JL, Deshane JS, Ryan AJ, Thannickal VJ, Carter AB. Macrophage Akt1 kinase-mediated mitophagy modulates apoptosis resistance and pulmonary fibrosis. Immunity. 2016;44(3):582-596.

13. Gieseck RL, Wilson MS, Wynn TA. Type 2 immunity in tissue repair and fibrosis. Nat Rev Immunol. 2018;18(1):62-76. 